\title{
INCERTIDUMBRE EN LA DISPONIBILIDAD DE RECURSOS HIDRICOS SUPERFICIALES EN LA CUENCA DEL EBRO: ANÁLISIS TEMPORAL DESDE UNA PERSPECTIVA PLURISECULAR
}

\author{
M. A. Saz Sánchez y S. M. Vicente Serrano
}

Departamento de Geografía y Ordenación del Territorio, Facultad de Filosofía y Letras, Universidad de Zaragoza. Pedro Cerbuna 12, 50009 Zaragoza

masaz@posta.unizar.es

\begin{abstract}
Resumen: A partir de las reconstrucciones dendroclimáticas de los totales anuales de precipitación de Capdella (Pirineo Centro-Oriental) y Pallaruelo de Monegros (sector central del Valle del Ebro), se analiza la evolución de la pluviometría en la Cuenca del Ebro desde el siglo XVI. Estas reconstrucciones evidencian el carácter oscilante de la precipitación en los dos ambientes climáticos. Las anomalías pluviométricas más importantes aparecen en los siglos XVI y XVII, durante la Pequeña Edad del Hielo, y en la segunda mitad del siglo XX. Posteriormente, las series dendroclimáticas se utilizan para evaluar la validez de algunos análisis probabilísticos utilizados como herramientas de gestión y planificación hidrológica.
\end{abstract}

Palabras clave: Dendroclimatología, evolución de las precipitaciones, Pequeña Edad del Hielo, análisis probabilísticos, planificación hidrológica, Cuenca del Ebro.

\begin{abstract}
Using dendroclimatic reconstructions of annual precipitation in Capdella (Central-Eastern Pyrenees) and Pallaruelo de Monegros (Central Ebro Valley), rainfall evolution in Ebro Valley since $16^{\text {th }}$ century is analyzed. These reconstructions show precipitation fluctuations in both climatic areas. The main anomalies occur in $16^{\text {th }}$ and $17^{\text {th }}$ centuries, during Little Ice Age, as well as in the second half of $20^{\text {th }}$ century. Furthermore, dendroclimatic reconstructions allow us to evaluate some probabilistic analysis applied in hydrologic management.
\end{abstract}

Keywords: Dendroclimatology, rainfall evolution, Little Ice Age, probabilistic analysis, hydrologic management, Ebro Basin.

\footnotetext{
* Recibido: 19-03-2003. Aceptado: 30-04-2003.
} 


\section{Introducción}

La pluviometría de la Cuenca del Ebro está caracterizada principalmente por su variabilidad y la incertidumbre en los volúmenes de precipitación. La presencia de periodos secos más o menos prolongados constituye un fenómeno habitual en la región (Ascaso et al., 1981; Cuadrat, 1989; Cuadrat, 1991; Pérez y Creus, 1994; Creus et al., 1995; Creus 1996), unos intervalos de déficit pluviométrico que han podido condicionar la organización territorial a lo largo de la historia.

En este escenario climático se ha producido en los últimos decenios un aumento creciente en la demanda de agua. La instalación de nuevos regadíos, la escasa modernización de las técnicas de riego, la progresiva industrialización del eje del Ebro, el crecimiento de la población y el aumento en el consumo medio de agua por habitante, contribuyen a que exista un difícil equilibrio entre los recursos hídricos disponibles y la demanda, convirtiéndose las sequías en un fenómeno que va más allá de lo climático para convertirse en un hecho socioeconómico de primer orden.

Fruto de la preocupación existente es el elevado número de trabajos desarrollados para analizar la pluviometría de la Cuenca del Ebro en general y el fenómeno climático de las sequías en particular (entre otros Creus, 2001; Cuadrat et al., 2001; Vicente y Cuadrat 2001). Estas investigaciones señalan la importancia de las sequías en esta zona y su recurrencia, así como su incidencia sobre la conservación de los ecosistemas naturales (Pedrocchi, 1998) y la productividad de los cultivos (Austin et al., 1998).

Una parte de los esfuerzos realizados para estudiar la pluviometría y el fenómeno de las sequías se ha centrado en el desarrollo de análisis probabilísticos (Sadeguipour y Dracup, 1985; Hershfield, 1973 y 1981; Sivakumar, 1992). Estos trabajos analizan la posibilidad de ocurrencia de eventos extremos de precipitación de carácter húmedo o seco mediante métodos estadísticos paramétricos, basados en el estudio de las distribuciones de frecuencias de las series pluviométricas. Este tipo de análisis se utiliza también para desarrollar algunos aspectos de la planificación hidrológica, basando la previsión de recursos en función de los umbrales de probabilidad obtenidos, expresados a menudo en forma de frecuencia por medio del conocido como periodo de retorno o recurrencia (Beguería, 2002).

La información climática utilizada para estos trabajos proviene del Instituto Nacional de Meteorología o de las confederaciones hidrográficas, unos datos instrumentales que, aunque no libres de problemas (como más comunes la ausencia de 
registros en tramos más o menos largos de las series y su frecuente falta de homogeneidad), nos proporcionan una buena imagen del acontecer pluviométrico de los últimos 50 años en buena parte de la región y de todo el siglo XX e incluso de la segunda mitad del XIX en algunos observatorios.

Los resultados obtenidos con los registros instrumentales de precipitación son estadísticamente apropiados para el intervalo temporal utilizado. Para poder extrapolarlos hacia el futuro, con objeto de utilizar esa información probabilística como herramienta de gestión y planificación hidrológica, se asume que los patrones generales que rigen la pluviometría de la zona analizada van a mantenerse estables en las sucesivas décadas. Pero las caracteristicas del clima de un espacio geográfico no son un fenómeno inmutable del paisaje. La presencia de anomalías de diferente intensidad y de una duración media de entre un decenio y tres o más décadas, así como de periodos de cierta estabilidad frente a otros de elevada variabilidad, son a grandes rasgos los patrones que rigen el comportamiento del clima en el periodo climático actual, conocido como Subatlántico, que se extiende desde aproximadamente el 2500 B.P. (Lamb, 1972; Gribbin, 1979; Font Tullot, 1988). Además de esas variaciones, aparecen episodios más largos, seculares o pluriseculares, en los que las condiciones generales son más húmedas o secas y en los que es más o menos frecuente la aparición de anomalías.

Teniendo en cuenta este carácter variable del clima a distintas escalas temporales, se hace a nuestro juicio necesaria la obtención de fuentes de información más largas que aporten datos sobre la evolución plurisecular de las precipitaciones. Estas series de datos posibilitarian conocer la cronología y magnitud de las principales anomalías pluviométricas acaecidas en las últimas centurias y la posible excepcionalidad del momento climático actual, en una atmósfera fuertemente intervenida por el hombre. Pero además, estas series pluviométricas más largas nos permitirían comprobar si los análisis probabilísticos comúnmente utilizados para cuantificar la posibilidad de ocurrencia de un determinado evento o volumen de precipitación, ofrecen resultados estables en el tiempo o si, por el contrario, los niveles de probabilidad muestran variaciones temporales que elevan la incertidumbre sobre su fiabilidad como descriptores del clima más allá del periodo a partir del que se han calculado.

En definitiva, la posibilidad de disponer de fuentes de información de mayor amplitud temporal nos ha de permitir conocer de forma más precisa un hecho tan característico de cualquier paisaje como es la pluviometría, con importantes implicaciones socioeconómicas en un área geográfica que, como el Valle del Ebro, depende en buena medida de este variable recurso para su desarrollo y planificación. 


\section{Objetivos}

Dentro de este contexto temático, el objetivo que se pretende alcanzar con este trabajo es doble:

- Conocer la evolución plurisecular de los volúmenes anuales de precipitación en distintos puntos de la Cuenca del Ebro representativos de ambientes climáticos diferentes e importantes desde el punto de vista de la planificación hidrológica de esta zona.

- Evaluar en esos ambientes climáticos la fiabilidad que a medio y largo plazo tienen las aproximaciones probabilísticas que usualmente se aplican a las series de precipitación, estudiando su evolución temporal a lo largo de los siglos.

\section{Fuentes de información y metodología}

\subsection{Las reconstrucciones dendroclimáticas}

Para cubrir los objetivos propuestos, se han utilizado las series dendroclimáticas de la precipitación total anual de Capdella (Lérida) y Pallaruelo de Monegros (Huesca), reconstruidas a partir de la información instrumental registrada en sus estaciones meteorológicas y varias cronologías construidas en el cuadrante nororiental de España.

La dendroclimatología es uno de los métodos que mejores resultados ofrece en la reconstrucción del clima del pasado, en particular en el contexto temporal del último milenio (Jones et al., 1998). Su finalidad es extraer la información climática que contienen las series de crecimiento anual de las especies leñosas en las latitudes templadas (cronologías), reconstruyendo el clima del pasado en función de la respuesta actual de la planta a las variaciones del clima (Creus et al., 2000). Esta disciplina individualiza y analiza la relación entre el anillo anual y las variables climáticas. La decodificación de ese registro constituye una de las bases de las reconstrucciones, puesto que conocida esa relación en el presente es posible extrapolarla hacia el pasado.

Las reconstrucciones dendroclimáticas se basan en el principio de que el crecimiento radial de las especies leñosas está condicionado fundamentalmente por el clima, hasta el punto de minimizar los efectos de otros parámetros que también tienen capacidad para regular ese crecimiento (Fritts, 1976). Estos parámetros, considerados como ruido no climático, son eliminados durante el desarrollo metodológico de 
las reconstrucciones (la metodología tradicional puede consultarse entre otros en: Fritts, 1976; Guiot, 1990; Fritts, 1991; Cook y Kairiukstis, 1990), definiendo algunos autores el anillo anual como el lugar en el que la planta codifica los impulsos procedentes de su entorno ambiental, en particular los climáticos (Corona, 1990).

La ventaja fundamental de la dendroclimatología frente a otras fuentes de información paleoclimática es que proporciona datos de carácter cuantitativo de la precipitación (en mm) y las temperaturas $\left(\mathrm{en}^{\circ} \mathrm{C}\right.$ ) de las pasadas centurias, una información continua en el tiempo, de correcta datación absoluta y de una resolución temporal cuando menos anual, con la única limitación que supone la longevidad de los árboles utilizados para generar las cronologías, fuente de información fundamental junto a los datos instrumentales para el proceso de reconstrucción dendroclimática.

\subsection{Selección de observatorios y reconstrucción de los totales anuales de precipitación}

Los observatorios instrumentales reconstruidos se seleccionaron por disponer de registros fiables (libres de cualquier efecto urbano, de una longitud de al menos 40 años y con un porcentaje de lagunas de información escaso) y por estar situados en ámbitos climáticos diferenciados de la Cuenca del Ebro e importantes desde el punto de vista de su planificación hidrológica.

El observatorio de Capdella se encuentra situado en el Pirineo Centro-Oriental, un área de notable importancia en lo que se refiere a la captación y almacenamiento de recursos hídricos superficiales para su utilización en los regadíos de la margen izquierda del Ebro. Está localizado a $1.485 \mathrm{~m}$ sobre el nivel del mar en la cabecera del río Flamisell, al sur del Parque Nacional de Aigües Tortes y Sant Maurici. Por su parte, Pallaruelo de Monegros se localiza en el sector central del Valle del Ebro, al norte de la Sierra de Alcubierre y a una altitud sobre el nivel del mar de $343 \mathrm{~m}$, en un espacio geográfico en el que la productividad por hectárea de la agricultura de secano está en buena medida condicionada por los volúmenes anuales de precipitación (Martí, 1992).

Las cronologías utilizadas para realizar las reconstrucciones han sido las de Aigües Tortes (Pinus uncinata) en Lérida, Pinobajo (Pinus syluestris L.), Trapa (Pinus sylvestris L.) e Ibonciecho (Pinus sylvestris L.) en Huesca, Larra (Pinus uncinata R.) en Navarra, Peñarroya (Pinus uncinata R.) y Bellena (Pinus nigra Arnold ssp. salzmannii -Dunal- Franco) en Teruel, además de las de Torretón (Pinus nigra A. ssp. salzmannii -Dunal- Franco) y Barranco del Infierno (Pinus nigra A. ssp. salzmannii Dunal- Franco) en la Cordillera Ibérica Conquense (figura 1). 


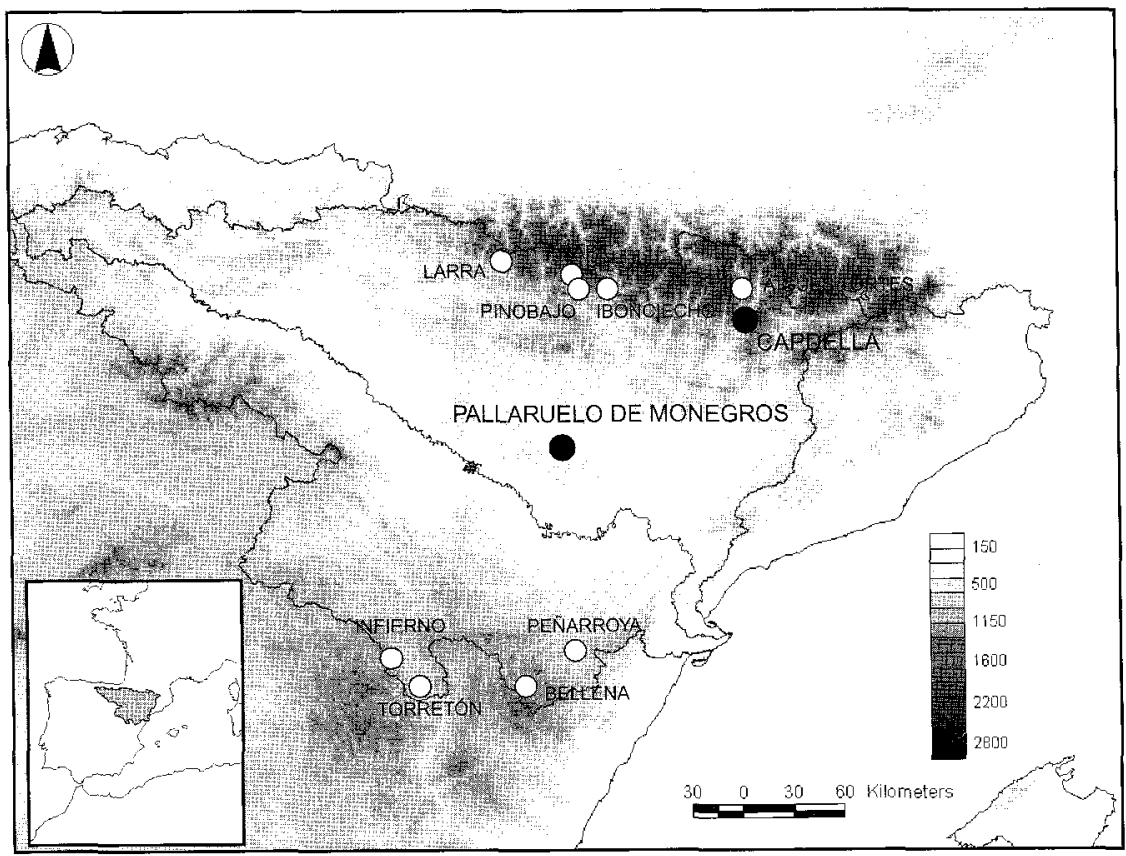

Figura 1. Localización de los observatorios reconstruidos y de las cronologías empleadas en el proceso de reconstrucción.

Una cronología es una serie temporal representativa del crecimiento radial de los árboles de una determinada zona o población forestal y constituye la base de información imprescindible, junto a los datos climáticos instrumentales, para cualquier intento de reconstrucción paleoclimática. Las utilizadas para estas reconstrucciones, fueron elaboradas durante la década de los años noventa con el soporte de varios proyectos de investigación nacionales y europeos dirigidos por el Dr. J. Creus (Instituto Pirenaico de Ecología, CSIC), quien además coordinó el proceso de reconstrucción de los totales anuales de precipitación de los observatorios seleccionados en este trabajo.

Estas cronologías fueron elaboradas a partir de las muestras obtenidas del crecimiento radial de los árboles de varios puntos del noreste español. En dendroclimatología, la selección de los ejemplares a muestrear no es aleatoria sino que se realiza teniendo en cuenta que éstos han de captar el máximo de señal climática posible, por lo que se seleccionan árboles situados cerca de sus límites altitudinales de distribución o sobre suelos pobres. Además, se seleccionan aquellos árboles que por su aspecto exterior indiquen una mayor edad. 
Los crecimientos anuales de los árboles seleccionados se midieron con un medidor ANIOL, de una precisión de $0,01 \mathrm{~mm}$, y posteriormente se dataron y sincronizaron con objeto de insertar los anillos ausentes o eliminar los falsos, procedimiento desarrollado con el programa COFECHA (Holmes, 1986). Estas series de crecimiento, tras ser estandarizas y transformadas en índices usando el programa ARSTAN, pueden agruparse para formar una cronología local independientemente de la edad de cada árbol. La reconstrucción de cualquier variable climática requiere de un mínimo de estas cronologías que dependerá de la tasa señal/ruido que contengan (Creus et al., 1995).

Para conocer la relación actual entre el crecimiento radial de los árboles, expresado en las cronologías, y el clima, se elabora la conocida como función respuesta, un modelo matemático que muestra con precisión qué variables han influido más sobre el crecimiento de los árboles.

Puesto que muchas de las variables climáticas que se utilizan suelen presentar una fuerte colinealidad (Creus y Beorlegui, 1996), en el cálculo de la función respuesta se emplea la regresión en componentes principales. Este método transforma las variables climáticas en una nueva serie de variables ortogonales denominadas autovectores. La varianza explicada por cada uno de los autovectores resultantes no es la misma en todos los casos y la regresión en componentes principales los ordena según sea el porcentaje de varianza explicada. Los que contribuyen en menor proporción a la explicación de la varianza total del sistema pueden haberse originado por errores de medición (Guiot et al., 1982; Génova, 1987), por lo que para subsanar esta deficiencia se procede a la calibración, verificación de los ajustes y validación del modelo multivariante para cada factor mediante técnicas bootstrap, añadidas a la investigación dendroclimática a comienzos de los años 90 (Fritts, 1990; Guiot, 1990; Cook y Kairiukstis, 1990). Este método supone un criterio muy estrecho de selección, pues sólo da validez a aquellos componentes que expliquen una elevada varianza. Con él, cada crecimiento de cada año se relaciona con 15 variables mensuales de temperatura, desde julio del año anetrior hasta septiembre del año en curso TJ-1 a TS, y otras tantas de precipitación (PJ-1 a PS).

El proceso se desarrolla con el programa PRECON, software desarrollado por el Tree-Ring-Laboratory de la Universidad de Tucson (Arizona), obteniéndose una ecuación final con todas las variables introducidas que toma la siguiente forma:

$$
I_{t}=C_{1}(T J-1)+C_{2}(T A g-1)+\ldots+C_{15}(T S)+C_{16}(P J-1)+\ldots+C_{30}(P S)+C_{31}(C r-1)+K
$$

en la que $I t$ es el crecimiento en el año $t$ que vendría explicado por la ecuación formada por el producto de los coeficientes $\left(\mathrm{C}_{1}\right.$ a $\left.\mathrm{C}_{30}\right)$ y las variables climáticas, desde la temperatura de julio del año anterior (TJ-1) hasta la precipitación de septiembre del año en curso (PS), correspondiendo el $\mathrm{C}_{31}$ con el crecimiento del año anterior. 

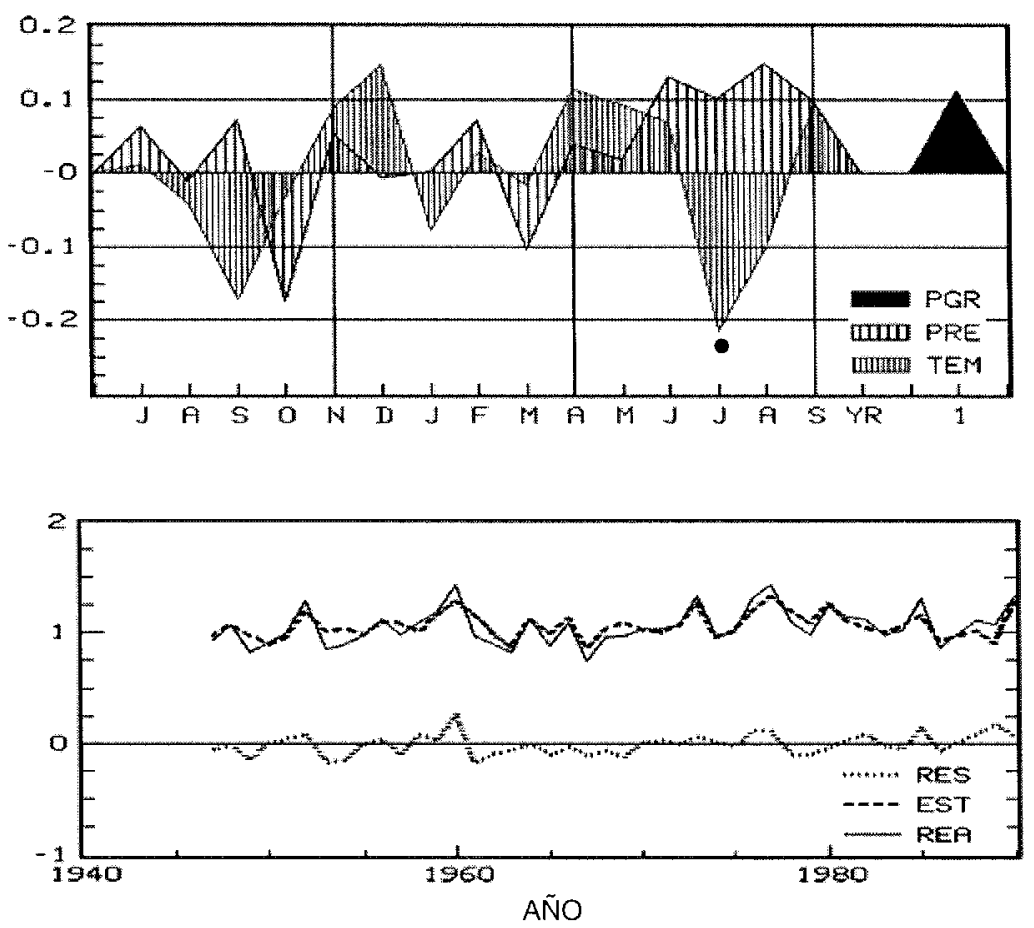

Figura 2. Ejemplo de función respuesta. Arriba: función respuesta de la cronología de Peñarroya (Pinus uncinata R.) con los datos instrumentales del observatorio de Pallaruelo de Monegros. Temperatura y precipitación: 1947-1990. Meses: julio año anterior a septiembre en curso. El punto negro indica que la relación es significativa.

Abajo: Valores reales (REA), estimados (EST) y residuales (RES) según el modelo calibrado entre los crecimientos de $P$. uncinata R. en Peñarroya y el observatorio de Pallaruelo de Monegros. $R^{2}=0,73$.

La función respuesta puede hacerse reversible y proceder en sentido contrario, para expresar finalmente el clima en función del crecimiento. Este segundo modelo es el que se conoce como función de transferencia, mediante la que las variables climáticas que la función respuesta señala que influyen en mayor medida en el crecimiento, aquéllas que han alcanzado mayor significación, podrán ser reconstruidas tantos años como longitud tengan las cronologías utilizadas para el cálculo de la función respuesta. En este caso, las cronologías actúan como predictores, mientras que las variables climáticas pasan a convertirse en variables dependientes.

En la última década se han realizado modificaciones en esta metodología (Fernández y Manrique, 1997) de manera que es posible utilizar conjuntamente un mayor número de cronologías, aunque éstas se encuentren a distancias espaciales importantes, siempre que tengan una elevada sincronización entre sí. Al intervenir un mayor número de pre- 
dictores en el proceso (cronologías), la señal climática reconstruible se incrementa de manera importante al depender de la que aportan conjuntamente.

\subsection{Valores pluviométricos reconstruidos}

Mediante esta metodología fue posible reconstruir en ambos observatorios los totales anuales de precipitación desde el año 1500. En la figura 3 se han representado gráficamente las series reconstruidas.

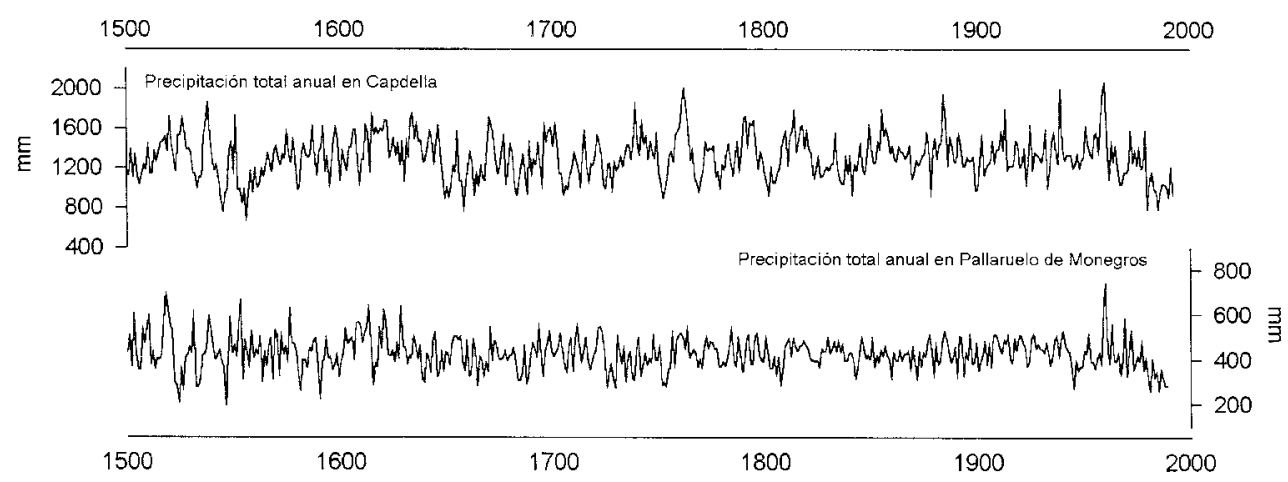

Figura 3. Series reconstruidas de los totales anuales de precipitación de Capdella y Pallaruelo de Monegros.

En cuanto a la representatividad de estas reconstrucciones como indicadores de la evolución de la pluviometría en el área pirenaica (en el caso de Capdella) y en el sector central del Valle del Ebro (en Pallaruelo de Monegros), ha de tenerse en cuenta que si bien las reconstrucciones necesitan de la información instrumental de un observatorio meteorológico tradicional para su calibración con las cronologías, la disposición geográfica de éstas y su propia naturaleza, hacen que la información reconstruida ofrezca una imagen de la evolución del clima de espacios geográficos más amplios que los observatorios instrumentales tradicionales, captando en mayor medida una señal macroclimática que, aunque sólo se aproxima a la precisión cuantitativa de la instrumental, muestra de una forma precisa las variaciones del clima en las pasadas centurias en escalas territoriales más amplias.

No obstante y con objeto de corroborar esa consideración espacial macroclimática de la información reconstruida, se ha analizado la relación existente entre las series 
de la precipitación total anual reconstruidas en Capdella y Pallaruelo de Monegros con series regionales de esa misma variable construidas a partir de datos instrumentales de varios observatorios de los Pirineos y del sector central del Valle del Ebro respectivamente (figuras 4 y 5 ).

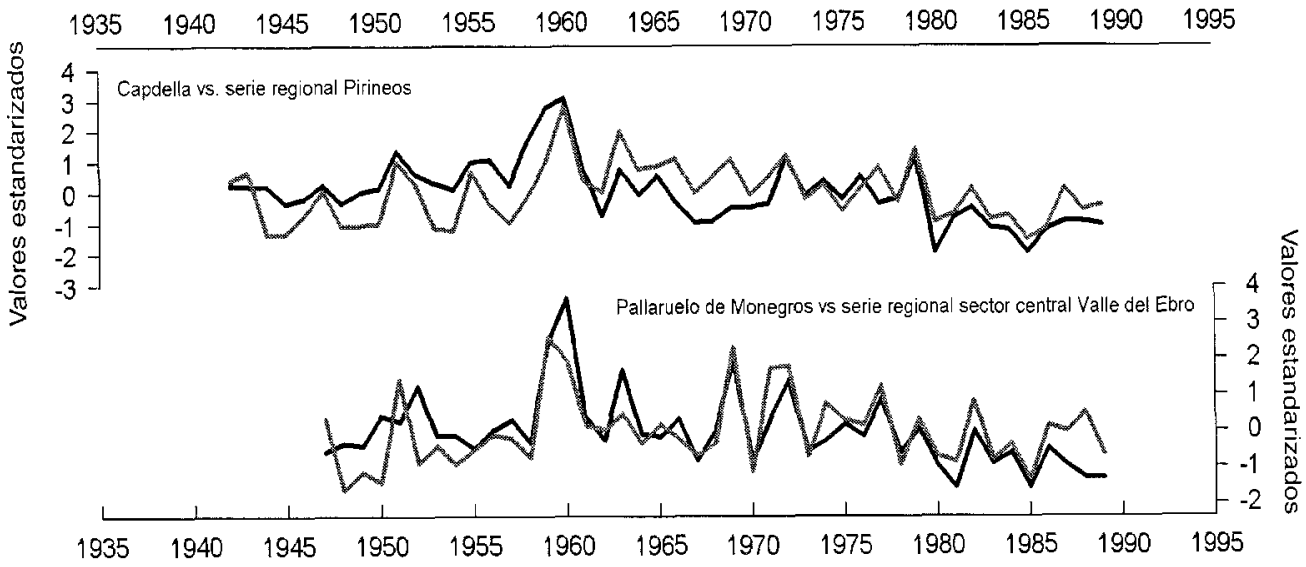

Figura 4. Comparación entre la información reconstruida en los observatorios de Capdella y Pallaruelo de Monegros (líneas negras) y las series regionales obtenidas para Pirineos y el sector central del Valle del Ebro (líneas grises).
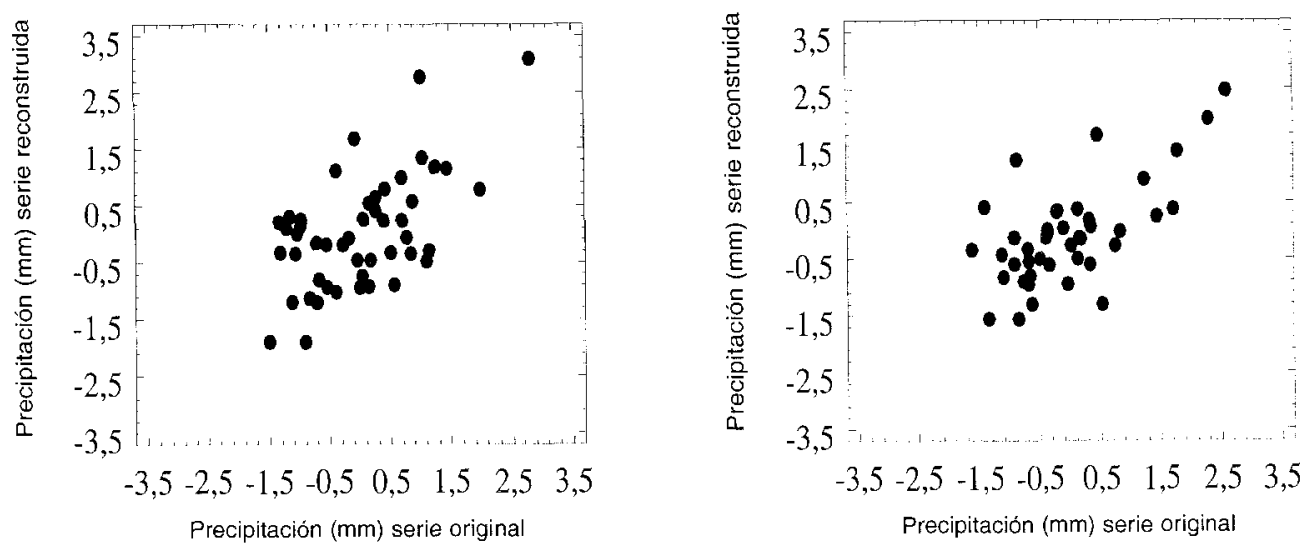

Figura 5. Relación entre la serie reconstruida en Capdella y la serie regional de Pirineos (izquierda) y la serie reconstruida en Pallaruelo de Monegros y la serie regional del sector central del Valle del Ebro (derecha). 
En el caso del observatorio pirenaico, la reconstrucción de Capdella se ha comparado con la serie regional estandarizada calculada a partir de los datos de la precipitación total anual de 21 observatorios instrumentales situados en las cuencas de los ríos Cincá, Ésera y Noguera Ribagorzana. La correlación entre ambas series es de 0,53, significativa $\alpha=0,01$.

En Pallaruelo de Monegros, los datos reconstruidos se han correlacionado con la serie regional estandarizada obtenida para el sector central del Valle del Ebro a partir de un conjunto de 41 observatorios de la zona. El coeficiente de correlación obtenido es algo mayor, de 0,68 , y también significativo $\alpha=0,01$. Ambos valores apoyarían la consideración de las dos reconstrucciones como representativas de la evolución de la precipitación total anual en el ámbito pirenaico y el sector central del valle del Ebro.

\subsection{Cálculo de los periodos de retorno}

A partir de las reconstrucciones de la precipitación total anual de estos observatorios, se va a tratar de evaluar la fiabilidad que a medio y largo plazo y en ambientes climáticos distintos, tienen las aproximaciones probabilísticas que usualmente se aplican a las series de precipitación, estudiando su evolución temporal a lo largo de los siglos.

La aplicación de análisis probabilísticos en los estudios sobre precipitación, se ha dirigido a menudo hacia la obtención de la probabilidad de ocurrencia de un valor de precipitación por debajo del cual se considera que un año es seco. La inversa de ese valor de probabilidad es el que se conoce como periodo de retorno, que no es sino el número de años esperado entre dos eventos de precipitación por debajo de ese umbral, o lo que en este caso es lo mismo, entre dos años secos.

\subsubsection{Determinación de los años secos}

Existen distintos métodos para categorizar un año como seco según su precipitación total: métodos basados en la distribución de frecuencias de las series de datos disponibles relacionadas con las desviaciones respecto a la media (McKee et al., 1993) o la mediana (Pita, 2001) y los utilizados más frecuentemente basados en el cálculo de percentiles (Gibbs, 1975; Gibbs y Mahler, 1967; Kinninmonth et al., 2000; Smith et al., 1993), para lo que es necesario asumir que la distribución de frecuencias de las precipitaciones anuales se ajustan a la distribución normal.

La determinación del umbral de precipitación a partir del cual podemos considerar un año como seco o muy seco mediante el uso de percentiles puede ser diverso. 
Gibbs y Maher (1967) consideran años extremadamente secos aquellos cuyos totales de precipitación se encuentran por debajo del percentil 10. Similar criterio utilizan Keyantash y Dracup (2002). En España, Almarza (2000) considera que el intervalo del $40 \%$ es representativo para identificar años deficitarios en cuanto al volumen de precipitación. Sin embargo, ese umbral no resulta a nuestro juicio adecuado para determinar unas condiciones claramente deficitarias de precipitación en las que los recursos hídricos se convierten en un claro limitante. Es por ello que en este trabajo se ha tenido en cuenta el valor del percentil 20 , umbral que ya puede considerarse como una frecuencia representativa de condiciones hídricas deficitarias.

\subsubsection{Obtención del periodo de retorno de un año seco}

A partir de las series dendroclimáticas calcularemos en este trabajo la evolución, en las últimas cinco centurias, de la probabilidad de ocurrencia y los periodos de retorno en años con precipitación inferior al valor del percentil 20, utilizando ventanas móviles de 50 años. De esta forma, el primer periodo considerado es el 1500-1549, el segundo incrementa un año y comprende el periodo 1501-1550, y así sucesivamente hasta 1940-1989.

Tras comprobar mediante el test de Kolmogorov (Watson, 1961; Pearson, 1963; Essenwanger, 1986) que las dos series de totales anuales reconstruidos siguen una distribución normal, podemos convertir la variable normal (n) en una normal estándar (U) de media 0 y desviación 1 mediante:

$$
u_{x}=\frac{n_{x}-\bar{n}}{\sigma_{n}}
$$

A partir de esta variable de distribución normal estándar podemos calcular el valor de probabilidad de cada registro de $\mathrm{n}\left\{\mathrm{n}_{1}, \mathrm{n}_{2}, \ldots, \mathrm{n}_{\mathrm{x}}\right\}$ mediante una aproximación polinómica (Abramovitz y Stegun, 1965):

$$
F(u)=1-f(u)(b 1 q+b 2 q 2+b 3 q 3+b 4 q 4+b 5 q 5)
$$

donde $\mathrm{F}(\mathrm{u})$ es la probabilidad acumulada de un determinado valor de la serie $\mathrm{U}\left\{\mathrm{u}_{1}, \mathrm{u}_{2}, \ldots, \mathrm{u}_{\mathrm{x}}\right\}$.

$$
q=\frac{1}{1+p u}
$$

$\mathrm{p}=0.2316419$ y $\mathrm{u}$ debe ser positivo de tal manera que $-\mathrm{u}=\mathrm{u}$.

$b_{1}=0.139381530$

$b_{2}=-0.356563782$ 
$b_{3}=1.781477937$
$b_{4}=-1.821255978$
$b_{5}=1.330274429$

f(u) se calcula según Abramovitz y Stegun (1965):

$$
f(u)=\left(a_{0}+a_{2} u^{2}+a_{4} u^{4}+a_{6} u^{6}+a_{8} u^{8}+a_{10} u^{10}\right)^{-1}+\varepsilon(u)
$$

$$
\begin{aligned}
& a_{0}=2.5052367 \\
& a_{2}=1.2831204 \\
& a_{4}=0.2264718 \\
& a_{6}=0.1306469 \\
& a_{8}=-0.0202490 \\
& a_{10}=0.0039132 \\
& F(-u)=1-F(u)
\end{aligned}
$$

A partir de la probabilidad de no excedencia ( $\mathrm{F}(\mathrm{x})$, que en el caso de una variable normal estándar como la aquí calculada correspondería a $\mathrm{F}(\mathrm{u})$ ) de un determinado valor de la variable $\mathrm{N}$, puede estimarse su periodo de retorno, considerado como el promedio de tiempo entre dos eventos de igual intensidad o magnitud, de tal forma que:

$$
T_{(x)}=\frac{1}{F(x \leq X)}
$$

donde $\mathrm{T}(\mathrm{x})$ es el periodo de retorno en años y $\mathrm{F}(\mathrm{x}<\mathrm{X})$ la probabilidad de que un año presente una precipitación igual o inferior a $\mathrm{X}$.

En este caso los valores de $n_{x}$ son constantes, correspondiendo a los valores del percentil 20 de toda la serie. Sin embargo, al considerar un cálculo móvil a lo largo del tiempo, lo que cambian son los parámetros de la distribución normal correspondientes a cada periodo de 50 años, es decir $\bar{n}$ y $\sigma_{n}$. De tal forma que el cálculo móvil supone repetir el proceso de cálculo de los periodos de retorno cada ventana de 50 años, siendo $\mathrm{n}_{\mathrm{x}}$ constante pero con valores de $\bar{n}$ y $\sigma_{n}$ distintos para cada lapso temporal.

3.4.3. Obtención del periodo de retorno de un valor de precipitación por debajo de la media

Pero si para el estudio del fenómeno de las sequías puede ser de particular interés la obtención de la probabilidad de ocurrencia de un evento seco, desde el punto de vista de la planificación hidrológica no lo es menor calcular el periodo de retorno en años de valores de precipitación total anual inferiores a la media de la serie. 
En este caso $n_{x}$ ya no es constante, sino que en periodos de 50 años, con sus correspondientes $\bar{n}$ y $\sigma_{n}$, se estima la probabilidad de ocurrencia de un determinado valor de precipitación por debajo de la media de la serie y sus periodos de retorno asociados:

$$
T_{(x)}=\frac{1}{F(x \leq X)}
$$

donde $\mathrm{T}(\mathrm{x})$ es el periodo de retorno en años y $\mathrm{F}(\mathrm{x}<\mathrm{X})$ la probabilidad de que un año presente una precipitación igual o inferior a diferentes valores por debajo de la media.

\section{Resultados}

\subsection{Análisis de las reconstrucciones}

Para el estudio de la evolución plurisecular de los totales anuales de precipitación en los observatorios de Capdella y Pallaruelo de Monegros, los datos obtenidos en las reconstrucciones, representados gráficamente en la figura 3, han sido transformados en diferencias respecto de la media del periodo de referencia 1850-1950.

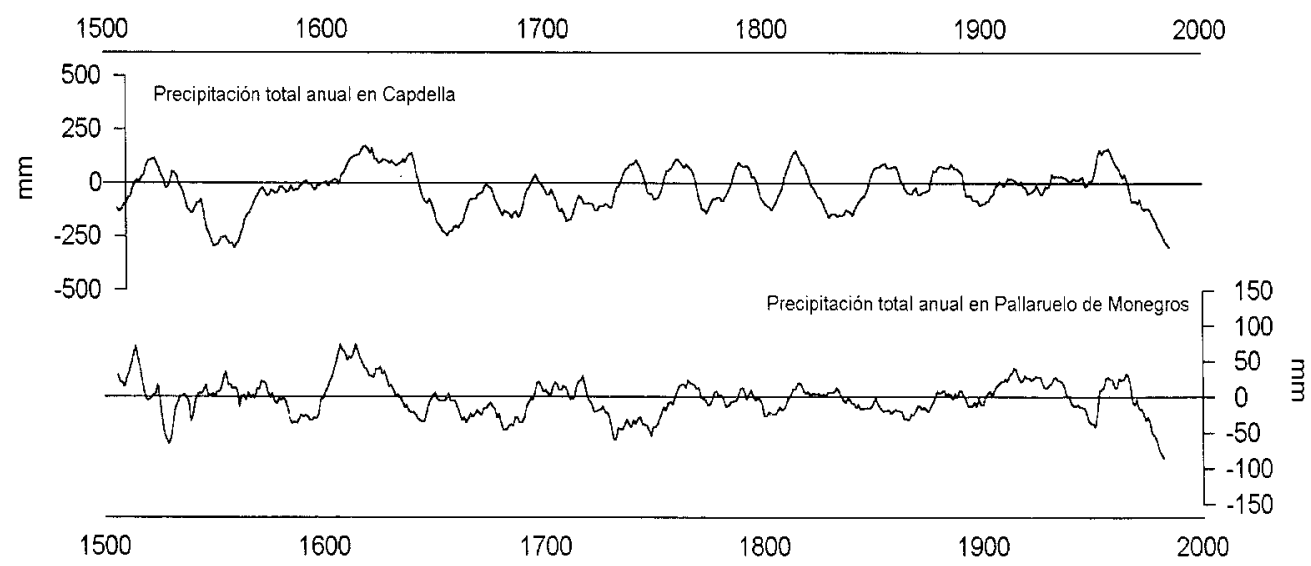

Figura 6. Series reconstruidas de los totales anuales de precipitación de Capdella y Pallaruelo de Monegros expresadas como diferencias respecto de la media del periodo 1850-1950 y suavizadas mediante media móvil de 15 años. 
Estos nuevos datos han sido representados en la figura 6, habiéndose aplicado previamente a las series un filtro de media móvil de 15 años. Este filtrado permite reducir la variabilidad natural de las series climáticas (Sneyers, 1992) con objeto de facilitar la detección de periodos anómalos. En la tabla 1 se señalan además los estadísticos principales obtenidos para estas dos series y los del periodo de referencia.

Tabla 1. Estadísticos principales de las series reconstruidas de la precipitación total anual en Capdella y Pallaruelo de Monegros, serie completa y periodo de referencia. LSCM: límite superior de confianza de la media; LICM: límite inferior de confianza de la media. Desv st: desviación estándar; Coef var: coeficiente de variación.

\begin{tabular}{|lcccc|}
\hline & \multicolumn{2}{c}{ Capdella } & \multicolumn{2}{c|}{ Pallaruelo de M. } \\
& $1500-1992$ & $1850-1950$ & $1500-1989$ & $1850-1950$ \\
\hline Media & 1313,6 & 1349,5 & 425,16 & 430,82 \\
LSCM & 1332,6 & 1384,9 & 432,22 & 442,03 \\
LICM & 1294,5 & 1314 & 418,1 & 419,60 \\
Varianza & 49309 & 32316 & 6493,3 & 3227,1 \\
Desv st & 222,06 & 179,77 & 80,58 & 56,81 \\
Mínimo & 666,6 & 922,00 & 131,9 & 268,30 \\
Máximo & 2066,8 & 1993,1 & 741 & 527,60 \\
Percentil 20 & 1128,4 & 1229,7 & 365,6 & 382,90 \\
Percentil 40 & 1251,2 & 1281,7 & 406,3 & 419,50 \\
Percentil 60 & 1358,8 & 1358,8 & 440,1 & 444,70 \\
Percentil 80 & 1484,7 & 1462,3 & 490,4 & 484,70 \\
Coef var & 16,91 & 13,32 & 18,95 & 13,19 \\
\hline
\end{tabular}

La elección del periodo 1850-1950 como periodo de referencia, se justifica por ser un intervalo temporal que queda fuera de la cronología tradicionalmente asignada en Europa Occidental para la Pequeña Edad del Hielo (Gribbin y Lamb, 1979; Jacoby y D'arrigo, 1989; Jones et al., 1998; Mann et al., 1998) y de las tendencias climáticas más recientes (Houghton et al., 1996; Houghton et al., 2002). Los análisis preliminares realizados sobre la información dendroclimática reconstruida en los observatorios utilizados en este trabajo (Creus y Saz, 1999; Saz y Creus, 1999) señalan además para el periodo 1850-1950 unos niveles de variabilidad en general moderados en el contexto de las últimas centurias y en cualquier caso inferiores a los observados en el periodo 1961-90, usualmente empleado como de referencia para la comparación entre medias, lo que junto a una cronología que quedaría fuera de la de los episodios climáticos más notables de nuestro milenio, lo hacen a nuestro juicio más aconsejable que el comúnmente utilizado 1961-90.

\subsubsection{Evolución de los totales anuales de precipitación en Capdella}

Comenzando el análisis sobre la evolución de la precipitación con el observatorio reconstruido en el ámbito pirenaico, destaca en la serie reconstruida la presencia en 
el tercio central del siglo XVI de un largo periodo seco, que se configura como la crisis pluviométrica seguramente más importante en esa zona en el marco temporal de las últimas cinco centurias. En concreto, en el periodo 1540-1568 la precipitación total anual media es inferior en un 18\% al promedio de referencia, destacando los descensos del $35 \%$ de los intervalos $1544-1547$ y $1552-1557$

Tras un periodo de más estabilidad de las precipitaciones en el tercio final del XVI y en los primeros años del XVII, buena parte de la primera mitad del XVII es más húmeda, con registros que en general superan el umbral de referencia. Sin embargo, la segunda mitad es de nuevo seca, destacando las prolongadas crisis pluviométricas de los intervalos 1648-1668 y 1678-1692, en que los descensos medios rondan el 15\%.

Este periodo más seco que marca en el área pirenaica buena parte de la segunda mitad del siglo XVII, se extiende al primer tercio del XVIII. El tercio central de esta centuria es por el contrario más húmedo, aunque en las últimas décadas aparecen nuevas crisis secas, como las de los intervalos $1767-1771$ y 1777-1781, con descensos medios cercanos al 20\% en ambos casos.

A lo largo del siglo XIX se detectan también algunas anomalías importantes, si bien no de la magnitud de las detectadas en las centurias precedentes. Las más notables suceden en la primera mitad, unas décadas en las que son mayoría los totales anuales de precipitación inferiores a la media de referencia. La segunda mitad de siglo es más lluviosa, pudiendo señalarse los intervalos 1853-1860 y 1880-1892.

A lo largo del XX destacan las anomalías detectadas en la segunda mitad de siglo. En la primera, la pluviometría no presenta oscilaciones importantes. Sin embargo, tras una década de los años 50 especialmente lluviosa, los volúmenes anuales de precipitación caen en el área pirenaica hasta el final de la serie reconstruida en valores mayoritariamente inferiores a la media de referencia, una crisis pluviométrica que la información dendroclimática señala como la más importante en las últimas centurias desde la descrita para el tercio central del siglo XVI, destacando el descenso medio del $27 \%$ del intervalo $1980-1992$.

\subsubsection{Evolución de los totales anuales de precipitación en Pallaruelo de Monegros}

La reconstrucción de los totales anuales de precipitación de Pallaruelo, representativa de la evolución de esta variable en el sector central del Valle del Ebro desde el siglo XVI, muestra también oscilaciones importantes, si bien no siempre coincidentes en cuanto a signo y cronología con las detectadas en el observatorio pirenaico.

El siglo XVI comienza con dos décadas húmedas, destacando los intervalos 1507 1510 y $1517-1521$, en los que los incrementos medios son del $26 \%$ y $38 \%$. En el resto 
de la década de los años veinte y durante la de los treinta, los volúmenes anuales son más bajos, con descensos medios del 25\% en los intervalos 1522-1528 y 1532-1536. Las décadas siguientes, frente a la crisis seca señalada en Capdella, no ofrecen en Pallaruelo anomalías importantes, aunque los totales anuales de precipitación, lejos de quedar en valores cercanos al de referencia, registran valores extremos. En el tramo final del siglo cabría destacar la crisis seca del 1580-1600, dos décadas en la que la precipitación total anual media es inferior en un 10\% a la del periodo de referencia $1850-1950$.

Tras esta anomalía de carácter seco, los totales anuales de precipitación reconstruidos en las primeras décadas del siglo XVII se muestran más generosos, coincidiendo con un periodo también más húmedo en Capdella. Sin embargo, y al igual que también sucedía en los Pirineos, la segunda mitad del siglo XVII es más seca, con descensos respecto del periodo de referencia del 11\% en los intervalos 1638-1651, $1657-1669$ y $1674-1689$.

Durante los primeros años del siglo XVIII los registros superan en general a la media de referencia, si bien en los años veinte, treinta y cuarenta y en los primeros años de la década de los cincuenta, se asiste a una nueva crisis seca, con un descenso medio de la precipitación total anual del 12,2\% entre 1725 y 1755.

La segunda mitad del siglo XVIII es más húmeda, aunque los totales de precipitación no se alejan demasiado de la media de referencia. Esta situación de menor variabilidad interanual de los totales de precipitación, se mantiene a lo largo de toda la primera mitad del XIX, un siglo que no ofrece anomalías comparables a la de los siglos anteriores y en el que únicamente destacaría el intervalo 1837-1882 por ofrecer registros que mayoritariamente quedan por debajo de la media de referencia.

En buena parte de la primera mitad del siglo XX son mayoría por el contrario los valores que superan ese umbral. Destaca después, en esta centuria, la crisis seca de la década de los cuarenta y, como en el observatorio de Capdella, la más húmeda década de los cincuenta y el notable descenso de las precipitaciones en el tramo final de la serie reconstruida, en una crisis que la reconstrucción del observatorio de Pallaruelo de Monegros la señala como la más importante en el sector central del Valle del Ebro al menos desde los siglos XVII y XVIII, con un descenso medio superior al 25\% en el intervalo $1975-1989$.

\subsubsection{Aspectos principales de la evolución de la pluviometría en la Cuenca del Ebro} desde el siglo XVI

El análisis realizado sobre las reconstrucciones de los totales anuales de precipitación de Capdella y Pallaruelo señalaría que, a una escala temporal plurisecular, la 
presencia de anomalías secas así como de otras de carácter húmedo son hechos habituales en la Cuenca del Ebro, sin que parezca existir una duración, magnitud y periodicidad medias de estos fenómenos.

La duración de las anomalías más importantes oscila entre menos de un decenio a más de tres décadas, y resulta a nuestro juicio especialmente significativa la magnitud de las crisis detectadas en la segunda mitad del siglo XX, comunes en cuanto a signo y cronología en las dos reconstrucciones, sobre todo por producirse tras un periodo de algo más de un siglo (siglo XIX y primeras décadas del XX) en el que las anomalías son de menor magnitud que en las décadas más recientes o que en los siglos XVI a XVIII.

Esta fase más estable coincide con las fechas en las que los estudios sobre la evolución del clima en el último milenio señalan el final de la Pequeña Edad del Hielo PEH- (Gribbin y Lamb, 1979; Jacoby y D'arrigo, 1989; Barriendos, 1994; Jones et al., 1998; Mann et al., 1998), el episodio climático más importante de las últimas centurias. El inicio de la PEH se situaría en el tramo final del siglo XV (Lamb, 1972; Gribbin y Lamb, 1979; Lockwood, 1979) y su dinámica interna se caracterizó, al menos en la Europa Occidental, por un descenso generalizado de las temperaturas (Jones, et al., 1998; Crowley, 2000; Luterbacher et al., 2000; Briffa et al., 2001), superior a $1^{\circ} \mathrm{C}$ en periodos de entre una y tres décadas, además de alteraciones en el régimen en las precipitaciones (Gribbin, 1979), manifestadas por la presencia de periodos secos y húmedos importantes (Bradley y Jones, 1992; Lamb, 1977).

Las fases más álgidas se centran en los siglos XVI y XVII, en especial en el tramo final del siglo XVII coincidiendo con el conocido como Mínimo Maunder (Brazdil et al., 1994; Briffa, 1994; Pfister, 1994; Luterbacher et al., 2000; Luterbacher et al., 2001), si bien se han constatado diferencias espaciales en cuanto a la cronología y características de los momentos de mayor intensidad de este episodio (Saz, 2003).

En el análisis de las reconstrucciones hemos visto que los siglos XVI y XVII albergarían también en el caso de la Cuenca del Ebro las anomalías pluviométricas más importantes de las últimas centurias. La segunda mitad del siglo XX sería comparable en el marco temporal analizado con la situación que muestran esas fases más intensas de la $\mathrm{PEH}$, habiéndose producido en las últimas décadas una variación en el escenario climático al que asistimos en la Cuenca del Ebro desde esa fase anterior más estable, el siglo XIX y la primera mitad del XX, en la que de forma progresiva terminaría la PEH, a la elevada variabilidad de la actualidad.

La particularidad que subyace en esta variación de escenario es que se produce en un momento en el que la atmósfera se encuentra altamente intervenida por al actividad antrópica, existiendo indicios de que el hombre ha podido alterar, al menos regionalmente, el comportamiento habitual de la atmósfera. 


\subsection{Las variaciones en la amplitud de los periodos de retorno a lo largo de los últimos cinco siglos en los Pirineos y en el sector central del Valle del Ebro}

La presencia de anomalías pluviométricas importantes a lo largo de los últimos siglos en los dos ambientes climáticos estudiados, con una duración media de entre menos de un decenio a tres o más décadas, y la posible excepcionalidad de la pluviometría actual en el marco temporal analizado, parecen poner en evidencia la consideración estacionaria que se aplica a las condiciones medias del clima de un espacio geográfico determinado.

A partir de esa condición, se consideran como herramientas válidas de gestión y planificación hidrológica los análisis probabilísticos realizados con información climática instrumental sobre la posibilidad de ocurrencia de un determinado evento de precipitación.

En este apartado analizaremos los resultados obtenidos tras aplicar estos métodos a series de datos pluviométricos de mayor longitud, la que posibilitan las series dendroclimáticas reconstruidas en los Pirineos (Capdella) y en el sector central del Valle del Ebro (Pallaruelo de Monegros), lo que ha de servirnos para discutir o al menos evaluar la validez de estos análisis probabilísticos como descriptores del clima a una escala temporal plurisecular.

\subsubsection{Evolución plurisecular de la amplitud del periodo de retorno de un año seco}

En la figura 7 se han representado gráficamente los resultados del cálculo de los periodos de retorno correspondientes al percentil 20 en Capdella y Pallaruelo de Monegros en sucesivas ventanas móviles de 50 años. En el caso del observatorio pirenaico el umbral delimitado por este percentil es de $1.128,4 \mathrm{~mm}$ y en Pallaruelo de $365,6 \mathrm{~mm}$, valores por debajo de los cuales podemos considerar un año como seco.

En ambos casos destacan las notables variaciones que a lo largo de las últimas cinco centurias muestra la amplitud del periodo de retorno de un año seco, con intervalos en los que superan los 20-22 años, frente a otros en los que es de entre $2,5 \mathrm{y}$ 3 años.

La primera mitad del siglo XVII, prácticamente la totalidad del XIX y la primera mitad del siglo XX, son los intervalos en los que la probabilidad de ocurrencia de años secos es más baja, ofreciendo los dos observatorios periodos de retorno más amplios para valores de la precipitación total anual por debajo del percentil 20.

El primero de esos intervalos coincide con la anomalía de carácter húmedo que en las primeras décadas del siglo XVII registran tanto el área pirenaica como el sec- 
tor central del Valle del Ebro, mientras que el siglo XIX y las primeras décadas del XX se caracterizan por la ausencia de anomalías secas o húmedas importantes y por ofrecer los niveles de variabilidad interanual de los volúmenes de precipitación más bajos en al menos las últimas cinco centurias (Saz, 2003). Estos dos aspectos son los que parecen condicionar la mayor amplitud del periodo de retorno de un año seco, en los dos ámbitos climáticos analizados, durante ese periodo de más de un siglo.

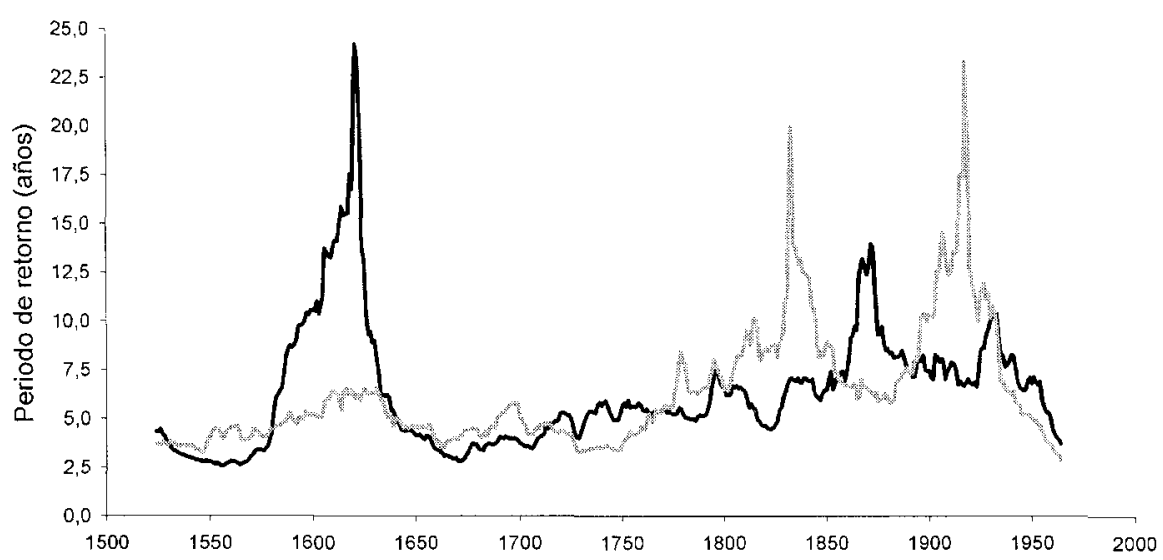

Figura 7. Evolución de los periodos de retorno del valor de precipitación correspondiente al percentil 20 calculado de forma secuencial en sucesivas ventanas de 50 años a partir de las series reconstruidas de la precipitación total anual de Capdella (línea negra) y Pallaruelo de Monegros (línea gris).

Frente a estas dos fases, la segunda mitad de los siglos XVI y XVI y las últimas décadas del XX, es decir, los momentos de mayor intensidad de la PEH a nivel continental en el primer caso y el periodo climático actual en el segundo, constituyen dentro de las últimas cinco centurias los intervalos en los que la posibilidad de ocurrencia de un año seco es mayor, destacando la tendencia a acortarse su recurrencia en las últimas décadas tanto en los Pirineos como en el sector central del Valle del Ebro.

La evolución temporal de la probabilidad de ocurrencia de eventos secos en las dos series pluviométricas reconstruidas, o lo que es lo mismo, en los dos ámbitos climáticos considerados parece ser, a la vista de la figura 7 , bastante similar.

Para cuantificar esta relación se ha utilizado el coeficiente de correlación de Spearman, un estadístico no paramétrico de características más robustas que los coe- 
ficientes paramétricos habitualmente aplicados ( $r$-Pearson), menos sensible a los extremos al fundamentar la correlación en el rango de los valores y para el que no es necesario asumir la normalidad de las series. El elevado valor que ofrece el coeficiente de correlación de Spearman obtenido entre las dos series, de 0,64 (significativo, $\alpha=0,01$ ), señalaría esa evolución común de la posibilidad de ocurrencia de un año seco en los dos observatorios.

En conjunto, en los dos observatorios la amplitud de los periodos de retorno de un año seco tiende a incrementarse desde la segunda mitad del XVII hasta el siglo XIX, mostrando este descriptor climático indicios de un progresivo final de las anomalías relacionadas con la $\mathrm{PEH}$, ya señalado para nuestras latitudes en otros trabajos realizados con información dendroclimática (Saz, 2003). En el siglo XIX y la primera mitad del XX, la evolución de la amplitud de los periodos de retorno muestra más diferencias entre las dos series, si bien en un contexto de menor probabilidad de ocurrencia de años secos que en las anteriores centurias, un hecho que podría relacionarse con ese carácter a menudo no generalizado de las anomalías pluviométricas en espacios geográficos incluso cercanos.

A pesar de estas diferencias observadas durante el siglo XIX y la primera mitad del XX, en los dos observatorios la segunda mitad del XX ofrece una acusada tendencia a acortar el periodo de retorno de los años secos frente al siglo y medio anterior, evidenciándose también a partir del cálculo secuencial de los periodos de retorno el señalado cambio de escenario climático, mostrando una posibilidad de ocurrencia de años secos sin precedentes desde la PEH.

4.2.2. Evolución plurisecular de la amplitud del periodo de retorno de diferentes valores de precipitación inferiores a la media

En la figura 8 se han representado los periodos de retorno en años para diferentes valores de precipitación anual en Capdella y Pallaruelo inferiores a la media de sus series, $1.313,6 \mathrm{~mm}$ y $425,16 \mathrm{~mm}$ respectivamente.

La metodología empleada para la obtención de un periodo de retorno es la misma que en el caso anterior, aunque calculándose los niveles de probabilidad dentro de intervalos temporales fijos de 50 años, desde la primera mitad del siglo XVI hasta la segunda mitad del XX.

En ambos casos vemos que los intervalos de medio siglo considerados, muestran periodos de retorno distintos para valores de precipitación total anual por debajo de la media de la serie. La segunda mitad del siglo XIX y la primera mitad del XX en los dos observatorios, así como también la primera mitad del XVII en Capdella y la primera del XIX en Pallaruelo, ofrecen periodos de retorno amplios para los valores de precipitación por 
debajo de la media más exiguos. En cambio, la segunda mitad del siglo XX, el siglo XVI, la segunda mitad del XVII y la primera del XVIII ofrecen recurrencias más cortas.

Las diferencias que en la probabilidad de ocurrencia de un mismo valor de precipitación se detectan entre distintos periodos son, en ocasiones, especialmente significativas. Sirva como ejemplo señalar que, en el sector central del valle del Ebro, la probabilidad de ocurrencia de valores por debajo de $275 \mathrm{~mm}$ en los intervalos 1801-1850, 1851-1900 y 1901-1950 es prácticamente nula, frente a intervalos como la primera mitad del XVI y la segunda mitad del XX en los que su recurrencia es inferior a los 20 años.
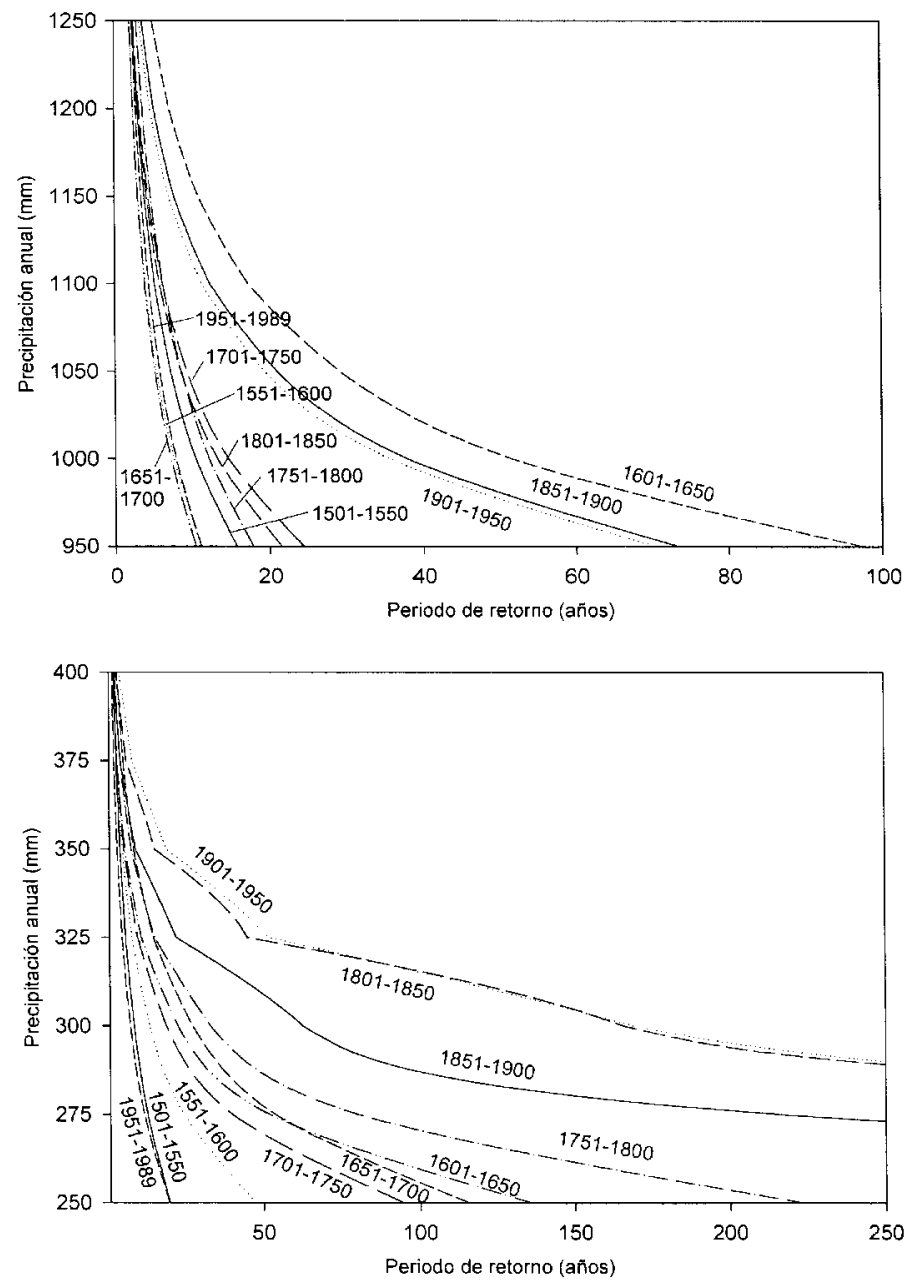

Figura 8. Periodo de retorno en años para diferentes valores de precipitación total anual por debajo de la media de las series en Capdella (arriba) y Pallaruelo de Monegros (abajo). 


\section{Discusión: incertidumbres en el cálculo de los periodos de retorno en un marco temporal plurisecular}

Estos resultados, tanto los obtenidos sobre la evolución temporal de la probabilidad de ocurrencia de un año seco, como en el cálculo de los periodos de retorno de distintos valores de precipitación total anual por debajo de la media, apuntarían en la misma dirección: a la mayor posibilidad de aparición de años secos en la Cuenca del Ebro en la segunda mitad del siglo XX y en las fases más álgidas de la PEH (siglos XVI y XVII), excepción hecha de la anomalía de carácter húmedo de la primera mitad del XVII, así como la menor probabilidad de ocurrencia de eventos secos en el XIX, en especial en la segunda mitad, y en la primera mitad del XX.

Pero además, estos resultados indicarían que, a una escala temporal pluridecenal, secular o plurisecular, este tipo de análisis ofrece diferencias notables y a nuestro juicio especialmente significativas en la probabilidad de ocurrencia de un evento seco en función del intervalo temporal en el que se han calculado. Unas diferencias tan importantes como las que señalarian para un mismo evento de precipitación un periodo de recurrencia inferior a 20 años o una posibilidad prácticamente nula de que éste se produzca.

La utilización de los periodos de retorno como herramientas de gestión y planificación puede resultar pues inadecuada, o cuando menos arriesgada, si a partir de ellas se trata de extrapolar hacia el futuro los resultados obtenidos en un periodo con unas características pluviométricas determinadas que, en la Cuenca del Ebro, no son durante los últimos cinco siglos constantes. Un cambio en esas condiciones medias altera, ya hemos visto que en ocasiones de forma notable, los resultados del análisis probabilístico y con ello la teórica frecuencia de aparición de años secos.

Esto genera, sin duda, un elevado grado de incertidumbre si lo que se pretende es utilizar como descriptores del clima a efectos de planificación unos datos sobre probabilidad de ocurrencia de determinados eventos de precipitación obtenidos en un contexto climático temporal determinado, que puede no tener unos patrones de comportamiento de las precipitaciones comunes con los de las décadas siguientes.

\section{Conclusiones}

El conocimiento de la precipitación en la Cuenca del Ebro, un fenómeno complejo y de fuertes implicaciones socioeconómicas, no puede quedar limitado al estudio de los datos instrumentales de los observatorios meteorológicos tradicionales. Es nece- 
sario disponer de fuentes de información y métodos de reconstrucción climática que, como la dendroclimatología, pueden aportar información de carácter cuantitativo sobre la evolución de las lluvias en las últimas centurias.

La información dendroclimática obtenida para este trabajo nos muestra la evolución de los totales anuales de precipitación desde el siglo XVI en dos espacios importantes desde el punto de vista de la planificación hidrológica de la Cuenca del Ebro: el Pirineo y el sector central. En ambos espacios, la presencia de anomalías secas y húmedas de una duración de entre menos de un decenio a tres o más décadas constituye un carácter esencial de su pluviometría, habiendo aumentado en las últimas décadas (segunda mitad del siglo XX) la intensidad de las anomalías, alcanzando una magnitud sin precedentes desde las fases más álgidas de la PEH (siglos XVI y XVII).

Se detecta así en la segunda mitad del siglo XX, un cambio de escenario climático en las tierras del Ebro, al menos en lo referido a las lluvias, desde una fase anterior más estable, a lo largo del siglo XIX (coincidiendo con el progresivo final de la PEH) y la primera mitad del XX, a la situación observada en el presente.

Las series dendroclimáticas de la precipitación total anual han permitido evaluar la validez como herramientas de gestión y planificación de los análisis probabilísticos utilizados para el cálculo de los periodos de retorno de años secos o de valores de precipitación por debajo de la media.

La disparidad de los resultados obtenidos sobre la amplitud de estos periodos según sea el intervalo temporal elegido para su cálculo, indicarían que existe un elevado grado de incertidumbre en estos métodos y que parecen resultar inadecuados para extrapolar sus resultados hacia las próximas décadas en un contexto climático caracterizado por la presencia de oscilaciones de escala no solo plurisecular sino también decenal.

\section{Agradecimientos}

Este trabajo forma parte de los proyectos de investigación financiados por la CICYT: "Precipitaciones y temperaturas de la mitad septentrional de España a partir del siglo XVII, reconstrucciones dendroclimáticas" (CLI96-1862), "La sequía en Aragón: tendencias climáticas seculares y patrones de cambio ambiental" (CLI99-098) y "Caracterización espacio-temporal de las sequias en el valle medio del Ebro e identificación de sus impactos" (BSO2002-02743). Se encuentra además enmarcado en el Programa de grupos de investigación consolidados del Gobiemo de Aragón (Grupo de Clima, cambio global y sistemas naturales). 


\section{Bibliografía}

Abramowitz, M. y Stegun, I.A. (1965): Handbook of Mathematical Functions. New York, Dover Publications.

Almarza, C. (2000): Variaciones climáticas en España. Época instrumental. En El campo, 137. El cambio climático, p. 69-84.

Ascaso, A. y Casals, M. (1981): Periodos secos y sequías en la depresión central del Ebro. Geograpbicalia, 11-12, p. 55-71.

Austin, R.B., Cantero-Martínez, C., Arrúe, J.L., Playán. E. y Cano-Marcellán, P. (1998): Yield-rainfall relationships in cereal cropping systems in the Ebro river valley of Spain. European Journal of Agronomy, 8, p. $239-248$.

Barriendos, M. (1994): Climatología histórica de Cataluña. Aproximación a sus características generales (ss. XV-XIX). Barcelona, Universidad de Barcelona: Departamento de Geografía Física y Análisis Geográfico Regional (Tesis doctoral inédita).

Beguería, S. (2002): Revisión de métodos paramétricos para la estimación de la probabilidad de ocurrencia de eventos extremos en Climatología e Hidrología: el uso de series de excedencias y su comparación con las series de máximos anuales. En Cuadrat, J.M., Vicente, S.M. y Saz, M.A. (eds.). La información climática como berramienta de gestión ambiental. Zaragoza, Universidad de Zaragoza, p 83-92.

Bradley, R.S. y Jones, P.D. (1992): Climate Since A.D. 1500. Londres, Routledge.

Brazdil, R., Dobrovolny, P., Chocolac, B. y Munzer, J. (1994): Reconstruction of the climate of Bohemia and Moravia in the period of 1675 to 1715 on the basis of writ- ten sources. En: Frenzel, B. (ed.) Climatic Trends and Anomalies in Europe 16751715. Estrasburgo, European Science Foundation, p. 109-121.

Briffa, K.R. (1994): Tree-ring evidence of northern Fennoscandian summer temperatures during the Maunder Minimum period. En: Frenzel, B. (ed.) Climatic Trends and Anomalies in Europe 1675-1715. Estrasburgo, European Science Foundation, p. 23-32.

Briffa, K.R., Osborn, T.J., Schweingruber, F.H., Harris, I.C., Jones, P.D., Shiyatov, S.G. y Vaganov, E.A. (2001): Low frequency temperature variations from a northern treering density network. Journal of Geopbysical Research, 106, p. 2.929-2.941.

Cook, E.R. y Kairiukstis L.A. (1990): Methods of dendrocbronology. Londres, Kluwer Academic Publishers, 394 pp.

Corona, E. (1990): Inconstanza del clima: fluttuazioni pregresse. En Susmel, L. ed., Principi di Ecologia. Padua, Cluep, p. 356-362.

Creus, J. (1996): Variaciones en la disponibilidad hídrica mensual en el valle del Ebro. En Marzol, M.V., Dorta, P. y Valladares, P. (eds.). Clima y agua: la gestión de un recurso climático. La Laguna, Universidad de La Laguna, p. 79-86.

Creus, J. (2001): Las sequías en el valle del Ebro. En Gil Olcina y Morales Gil (eds.) Causas y consecuencias de las sequías en España. Alicante, Universidad de Alicante, p. 231-260.

Creus, J. y Ferraz, J. (1995): Irregularidad pluviométrica y continentalidad térmica en el valle medio del Ebro. Lucas Mallada, 7 , p. 147-164. 
Creus, J., Beorlogui, M., Fernández, A. (1995): Reconstrucciones climáticas en Galicia durante las últimas centurias. Estudio Dendrocronológico. La Coruña, Presidencia de la Xunta de Galicia.

Creus, J. y Beorlegui, M. (1996): La multicolinealidad de los datos climáticos. La regresión en componentes principales. En Modelos y Sistemas de Información Geográfica. VII Coloquio de Geografia Cuantitativa. Universidad del País Vasco, p 38-47.

Creus, J. y Saz, M.A. (1999): Estudio de la variabilidad climática del último milenio a partir de series de temperatura y precipitación reconstruidas en el noreste español. En Raso, J.M. y Martín Vide, J. (eds.) La Climatología española en los albores del siglo XXI. Publicaciones de la Asociación Española de Climatología (AEC). Serie A, n. ${ }^{\circ}$, p. 155-164.

Creus, J., Fernández, A. y Manrique, E. (2000): Análisis de la variabilidad del clima español durante el último milenio. El Campo. 137, p. 27-48.

Crowley, T.J. (2000): Causes of Climate Change Over the Past 1000 Years, Science, 289 , p. $270-277$.

Cuadrat, J. (1989): Las oscilaciones climáticas recientes en Zaragoza (1865-1984). Geographicalia, 26, p. 53-60.

Cuadrat, J. (1991): La sequía en el valle del Ebro. Aspectos climáticos y consecuencias económicas. Revista Real Academia de Ciencias, 85, p. 537-545.

Cuadrat, J.M. (2001): Percepción de la sequía en la Fachada Cantábrica. En Gil Olcina, A. y Morales Gil, A. (eds.). Causas y consecuencias de las sequias en España. Alicante, Caja de Ahorros del Mediterráneo e Instituto Universitario de Geografía de la Universidad de Alicante. Alicante, p. 277-301.

Cuadrat, J.M., Creus, J., Vicente Serrano, S.M. y Ferraz, J. (2001): La sequía en Aragón a través de los registros históricos. En Peña, J.L. y Longares, L.A. (eds.) Aportaciones geográficas en memoria del prof. L. Miguel Yetano Ruiz, p. 123-130.

Essenwanger, O.M. (1986): Elements of Statistical Analysis. En Landsberg, H.E. (ed.) World Survey of Climatology. Londres, Elsevier, vol. 1.

Fernández, A. y Manrique, E. (1997): Nueva metodología para la reconstrucción dendroclimática y aplicaciones más importantes. Madrid, INIA.

Fritts, H. (1976): Tree rings and climate. Blackburn Academic Press, 567 pp.

Fritts, H. (1990): Modelling tree-ring and environmental relationships for dendrochronological analisys. En Dixon et al. (eds.) Forest growth process modeling of responses to environmental stress. Oregon, Timers Press, p 360-382.

Fritts, H. (1991): Reconstmucting large-scale climatic patterns from tree-ring data. Arizona, University of Arizona Press.

Font Tullot, I. (1988): Historia del clima de España. Cambios climáticos y sus causas. Madrid, Instituto Nacional de Meteorología.

Génova, R. (1987): Análisis y significado de los anillos de crecimiento de dos especies forestales: Pinus uncinata y Pinus sylvestris, en la Peninsula Ibérica. Barcelona, Universidad de Barcelona. 
Gibbs, W.J. y Maher, J.V. (1967): Rainfall Deciles as drought indicators. Australian Bureau of Meteorology Bull. 48, 37 pp.

Gibbs, W.J. (1975): Drought, its definition delineation and effects. En Lectures presented at the $26^{\text {th }}$ session of the WMO Executive Committe. Ginebra, Special Environmental Report no 5. WMO, p. 3-30.

Gribbin, J. (ed.) (1979): Climatic Changes. Cambridge, Cambridge University Press.

Gribbin, J. and Lamb, H.H. (1979): Climatic change in historical times En Gribbin, J. (ed.) Climatic Change. Cambridge University Press.

Guiot, J. (1990): Methods of calibration. En Cook, E.R. y Kairiukstis, L.A. (eds.) Metbods of dendrocbronology, Kluwer Academic Publishers, p. 165-172.

Guiot, J., Berger, A. y Munault, A. (1982): An illustration of alternative transfer function methods in Switzerland. En Hughes, M.K., Kelly, P.M., Pilcher, J.R. y Lamarche, V.C., Climate from Tree-Rings. Cambridge University Press. p. 160-162.

Hershfield, D.M. (1973): On the probability of extreme rainfall events. Bulletin of the American Meteorological Society. 54 , p. 1.013-1.018.

Hershfield, D.M. (1981): Frecuency of dry day sequences. Water Resources Bulletin. 17, p. 317-320.

Holmes, R. (1986): Dendrocbronology program library. Guide for computer program COFECHA. Arizona. Laboratory of Tree-Ring Research.

Houghton, J.R., Meira Filho, L.G., Callander, B.A., Harris, N., Kattenberg, A. y Maskell, K. (eds.) (1996): Climatic change 1995: the science of climate change, Cambridge, Cambridge University Press, p. 572.

Houghton, J.R., Ding, Y., Griggs, M., Noguer, P.J. Van Der Linden y Xiaosu, D. (eds.) (2001): Climate Change 2001: The Scientific Basis. Contribution of Working Group I to the Third Assessment Report of the Intergovernmental Panel on Climate Change (IPCC). Cambridge. Cambridge University Press, p. 944.

Jacoby, G.C. y D'arrigo, R. (1989): Reconstructed Northern Hemisphere annual temperature since 1671 based on high latitude tree ring data from North America. Climatic Change, 14, p. 39-59.

Jones, P.D., Briffa, K.R., Barnett, T.P. y Tett, S.F.B. (1998): High resolution paleoclimatic records for the last millenium: interpretation, integration an comparison with General Circulation Model control-run temperatures. The Holocene, 8 (4), p. 455-471.

Keyantash, J. y Dracup, J. (2002): The quantification of drought: an evaluation of drought indices. Bulletin of the American Meteorological Society, 83 (8), p. 1167-1180.

Kinninmonth, W.R., Voice, M.E., Beard, G.S., de Hoedt, G.C. y Mullen, C.E. (2000): Australian climate services for drought management. En Wilhite, D.A. (ed.) Drougbt: $A$ Global Assessment. Routledge, p. 210-222.

Lamb, H.H. (1972): Climate: present, past and future. Londres, Mathuen \& Co.

Lamb, H.H. (1977): Climate: present, past and future. Londres, Mathuen \& Co. Vol. II.

Lockwood, J.G. (1979): Causes of climate. Londres, Edward Arnold. 
Luterbacher, J., Rickli, R., Tinguely, C., Xoplaki, E., Schüpbach, E., Dietrich, D., Hüsler, J., Ambühl, M., Pfister, C., Beeli, P. Dietrich, U., Dannecker, A., Davies, T.D., Jones, P.D., Slonosky, V., Ogilvie, A.E.J., Maheras, P., Kolyva-Machera, F., MartínVide, J., Barriendos, M., Alcoforado, M.J., Nunes, M.F., Jonsson, T., Glaser, R., Jacobeit, J., Beck, C., Philipp, A., Beyer, U., Kaas, E., Schmith, T., Bärring, L., Jönsson, P., Rácz, L. y Wanner, H. (2000) Monthly mean pressure reconstruction for the Late Maunder Minimum period ( $A D$ 1675-1715). International Journal of Climatology, 20, p. 1049-1066

Luterbacher, J., Rickli, R., Xoplaki, E., Tinguely, C., Beck, C., Pfister, C. y Wanner, H. (2001): The late Maunder Minimum (1675-1715) - A key period for studying decadal scale climatic change in Europe. Climatic Change, 49, p. 441-462.

Mann, M.E., Bradley, R.S. and Hughes, M.K. (1998): Global-scale temperature patterns and climate forcing over the past six centuries. Nature, 392, p. 779-787.

Martí, A. (1992): La agroclimatología de los Monegros: alternativas al uso tradicional del secano. Huesca, Instituto de estudios Altoaragoneses.

McKee, T.B., Doesken, N.J. and Fleist, J. (1993): The relationship of drought frequency and duration to time scales. Eight Conference on Applied Climatology, p. 179-184.

Pearson, E.S. (1963): Comparison of tests for randomness of points of a line. Biometrika, 34, p. $123-169$.

Pedrocchi, C. (1998): Ecología de los Monegros. Huesca, Instituto de Estudios Altoaragoneses.
Pérez, J. y Creus, J. (1994): Tendencia secular de la precipitación en Zaragoza (18651984). En Pita, M.F. y Aguilar, M. (eds.) Cambios y variaciones climáticas en España. Sevilla, Universidad de Sevilla, p. 169-182.

Pfister, C. (1994): Spatial patterns of climatic change in Europe A.D. 1675-1715. En: Frenzel, B. (ed.) Climatic Trends and Anomalies in Europe 1675-1715. Estrasburgo, European Science Foundation, p. 287-316.

Pita, M.F. (2001): Sequías en la cuenca del Guadalquivir. En Gil Olcina, A. y Morales, A. (eds.) Causas y consecuencias de las sequias en España. Alicante, Universidad de Alicante, p. 303-344.

Sadeguipour, J. y Dracup, J.A. (1985): Regional frecuency analysis of hydrologic multiyear drought. Water Resources Bulletin, 21, p. 481-487.

Saz, M.A. (2003): Análisis de la evolución del clima en la mitad septentrional de España desde el siglo XV a partir de series dendroclimáticas. Zaragoza. Servicio de Publicaciones de la Universidad de Zaragoza.

Saz, M.A. y Creus, J. (1999): La variabilidad del clima español en el pasado: frecuencia de valores extremos de la temperatura y precipitación trimestrales reconstruidas desde el siglo XV. En Raso, J.M. y Martín Vide, J. (eds.) La Climatología española en los albores del siglo XXI. Madrid. Publicaciones de la Asociación Española de Climatología (AEC). Serie A, n. ${ }^{\circ}$, p. 501-510.

Sivakumar, M.V.K. (1992): Climate change and implications for agriculture in Niger. Climatic Change, 20, p. 297-312. 
Sneyers, R. (1992): Use and misuse of statistical methods for detection of climatic change. En Climate Change detection project, Report on the Informal Planning Meeting on Statistical Procedures for Climate Change detection. WDCDMP (20) WMO.

Smirth, D.I., Hutchinson, M.F. y McArthur, R.J. (1993): Australian climatic and agricultural drought: payments and policy. Drought Network News, 5, p. 11-12.
Vicente, S.M. y Cuadrat, J.M. (2001): Desarrollo de una metodología para la evaluación de la variabilidad espacial en la intensidad de los periodos secos: Aplicación en el valle medio del río Ebro. En Pérez-Cueva, A.J. López, E. y Tamayo, J. (eds.) El Tiempo del Clima. Barcelona, Asociación Española de Climatología, p. 255-267.

Watson, G.S. (1961): Goodnes of fit-test on a circle. Biometrika, 48, p. 109-114. 